\title{
The SenseEmotion Database: A Multimodal Database for the Development and Systematic Validation of an Automatic Pain- and Emotion-Recognition System
}

\author{
Maria Velana ${ }^{1}$, Sascha Gruss ${ }^{1}$, Georg Layher ${ }^{2}$, Patrick Thiam ${ }^{2}$, \\ Yan Zhang ${ }^{2}$, Daniel Schork ${ }^{3}$, Viktor Kessler ${ }^{2}$, Sascha Meudt ${ }^{2}$, \\ Heiko Neumann ${ }^{2}$, Jonghwa Kim ${ }^{3}$, Friedhelm Schwenker ${ }^{2}$, \\ Elisabeth André ${ }^{3}$, Harald C. Traue ${ }^{1}$, and Steffen Walter ${ }^{1(\bowtie)}$ \\ 1 University Clinic of Psychosomatic Medicine and Psychotherapy, \\ Medical Psychology, Ulm University, 89075 Ulm, Germany \\ steffen.walter@uni-ulm.de \\ 2 Institute of Neural Information Processing, Ulm University, \\ 89069 Ulm, Germany \\ 3 Department of Computer Science, Human-Centered Multimedia, \\ University of Augsburg, 86159 Augsburg, Germany
}

\begin{abstract}
In our modern industrial society the group of the older (generation $65+$ ) is constantly growing. Many subjects of this group are severely affected by their health and are suffering from disability and pain. The problem with chronic illness and pain is that it lowers the patient's quality of life, and therefore accurate pain assessment is needed to facilitate effective pain management and treatment. In the future, automatic pain monitoring may enable health care professionals to assess and manage pain in a more and more objective way. To this end, the goal of our SenseEmotion project is to develop automatic painand emotion-recognition systems for successful assessment and effective personalized management of pain, particularly for the generation $65+$. In this paper the recently created SenseEmotion Database for pain- vs. emotion-recognition is presented. Data of 45 healthy subjects is collected to this database. For each subject approximately $30 \mathrm{~min}$ of multimodal sensory data has been recorded. For a comprehensive understanding of pain and affect three rather different modalities of data are included in this study: biopotentials, camera images of the facial region, and, for the first time, audio signals. Heat stimulation is applied to elicit pain, and affective image stimuli accompanied by sound stimuli are used for the elicitation of emotional states.
\end{abstract}




\section{Introduction}

The proportion of people aged 65 and over increases constantly over the years. Pain is common among older population and can greatly impact older people's quality of life, their physical and psychological functioning and become a barrier to social inclusion. In clinical and care home settings, accurate assessment of pain is essential for successful pain management. A failure to recognize and treat pain can lead to health problems and the unacceptable suffering of elderly. For instance, the lack of sufficient pain management is associated with pathophysiological effects, such as increased blood pressure and heart rate [24]. In general, valid and reliable pain assessment is necessary to facilitate successful pain management without complications [12,20] and enhance quality of life in older adults.

To date, self-reporting is the standard method for assessing pain. However, self-reporting requires the capacity to comprehend the task and to communicate about the experienced pain [32]. This implies that self-report scales are not always a valid and reliable tool for assessing pain in older people, especially in those who have demonstrable cognitive impairment. Moreover, comprehensive pain assessment should be regularly repeated, particularly if the individual is not able to communicate with health care professionals. Previous research on automatic pain recognition is considerable and can be mainly classified into videoand biopotential-based approaches $[6,8,11,19,28,29]$. However, there is a relative scarcity of studies incorporating both approaches. To the best of authors' knowledge, the study by Walter et al. [27] was the first one that developed a database using biopotential and visual signals. A multimodal pain recognition system incorporating a set of different modalities such as biosignals (e.g., cardiac electrical activity, trapezius muscle activity, skin conductance and respiration), video signals (e.g., facial expressions, skeleton data and head pose) and paralinguistic information is becoming increasingly important for objective and accurate pain assessment. The goal of the SenseEmotion project is the development of an automatic pain- and emotion-recognition system for the successful assessment and effective personalized management of pain in older people. Thus, a multimodal dataset for pain- and emotion-recognition was developed based on physiological, video- and audio-signals. Heat pain was induced experimentally in different levels. In addition, affective image stimuli selected from the International Affective Picture System (IAPS) [16] and the Emotional Picture Set (EmoPicS) [30] were used to evoke positive, negative and neutral emotions. The image stimuli were accompanied by affective sound stimuli so as to intensify affective reactions that would be induced by the image stimuli.

The aim of the present study was to detect patterns of heat pain intensities under the influence of emotional stimuli. The SenseEmotion Database contains the below unique parameters:

- Highly computer-controlled pain stimulation

- Affective induction through emotional stimuli in a two-dimensional space determined by pleasure and arousal ratings 
- Physiological measures - i.e., skin conductance level (SCL), electrocardiography (ECG), electromyography (EMG) and respiration (RSP)

- Multiple camera setup

- Depth map video from a Microsoft Kinect V2 with integrated microphone

- Digital wireless headset microphone in combination with a directional microphone

\section{Methodology}

\subsection{Participants}

A total of 45 healthy subjects participated in the experiment and received an expense allowance. Participants were recruited through advertisements placed on the campus of the University of Ulm. All participants were fully informed of the study protocol and provided written informed consent for their participation at the beginning of the study. Subjects were excluded for being $<18$ years old, having neurological problems, psychiatric disorders, chronic pain, headache disorders, cardiovascular diseases, regular use of analgesic medication, or use of analgesic medication directly before the experiment. The study was conducted according to the ethical guidelines set out in the WMA Declaration of Helsinki (ethical committee approval was granted: 196/10-UBB/bal). The study protocol was approved by the ethics committee of the University of Ulm (Helmholtzstraße 20, 89081 Ulm, Germany).

\subsection{Design of the Experiment}

The Medoc Pathway thermal stimulator was employed to elicit pain [27]. ATS thermode of $30 \times 30 \mathrm{~mm}$ [22] was applied to the forearm of the participant (see Fig. 1). During the entire experiment, participants were seated in a chair with their arm resting on the desk in front of them. This system delivers precise painful and non-painful thermal stimuli [22] under highly controlled conditions without causing tissue damage [18]. Thermal stimuli temperatures range from $32^{\circ} \mathrm{C}$ to $55^{\circ} \mathrm{C}[22]$. During the entire experiment, stimuli temperature did not exceed $50.5^{\circ} \mathrm{C}[28]$.

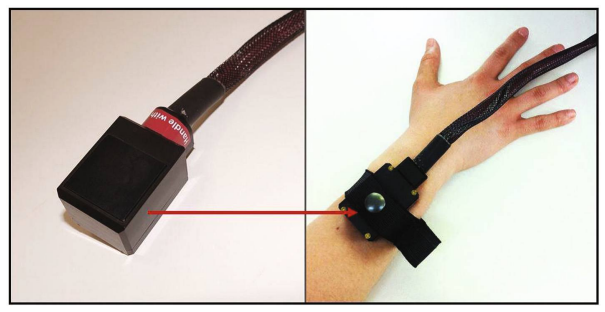

Fig. 1. Thermal pain stimulator that was applied to the participant's forearm. (Reprinted by kind permission of [5], p. 26) 
Heat Pain Calibration. At the beginning of the experimental session the researchers determined individual pain threshold $\left(T_{1}\right)$ and pain tolerance threshold $\left(T_{3}\right)$ for each participant. Pain threshold indicates the temperature that the subject's perception alters from heat to pain. Subjects were instructed to press the stop button as soon as heat stimulus became painful: Please press immediately the stop button, when a feeling of burn, sting, drill, or draw appears in addition to the feeling of heat. Pain tolerance threshold indicates the temperature that the subject's perception alters from heat to pain and the level that pain becomes intolerable. Hence, subjects were instructed to press the stop button as soon as heat stimulus was barely tolerable: Please press immediately the stop button, when you cannot accept heat with regard to the feeling of burn, sting, drill, or draw any more. In order to measure thresholds $T_{1}$ and $T_{3}$, temperature was gradually raised $\left(1{ }^{\circ} \mathrm{C} / \mathrm{s}\right)$ with a starting value of $32{ }^{\circ} \mathrm{C}\left(T_{0}\right)$ (see the below section). The researchers performed four measurements for $T_{1}$ and $T_{3}$ respectively. An average value was calculated for $T_{1}$ and $T_{3}$ thresholds for each participant. Subsequently, the researchers calculated the mean value of $T_{1}$ and $T_{3}$ determining one additional individual level $T_{2}$ (see Fig. 2). After the calibration phase, Pathway software was calibrated with the three pain levels $\left(T_{1}, T_{2}\right.$ and $T_{3}$ ) separately for each participant for the main part of the experiment.

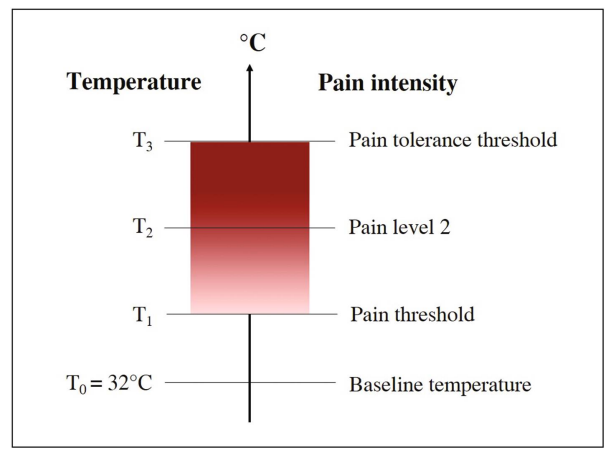

Fig. 2. Induced pain intensity depending on temperature. $T_{0}$ represents baseline temperature. $T_{1}, T_{2}$ and $T_{3}$ represent the three pain levels that were separately calculated for each participant.

Heat Pain Stimulation. During the main experimental phases each of the three individualized stimuli was randomly applied 30 times for approximately $30 \mathrm{~min}$, resulting in a total of 90 stimuli. The $T_{0}$ baseline temperature (no pain) was $32{ }^{\circ} \mathrm{C}$. Figure 3 displays a temperature plot of a stimulus and the subsequent pause. Each pain stimulus was performed for $4 \mathrm{~s}$. The pauses between the stimuli were randomized between $8-13 \mathrm{~s}$.

Affective Image Stimuli. 180 digital images were selected according to three levels of affective valence (highly pleasant, neutral and highly unpleasant) and two levels of arousal (low, high). During each experimental phase 30 pleasant 


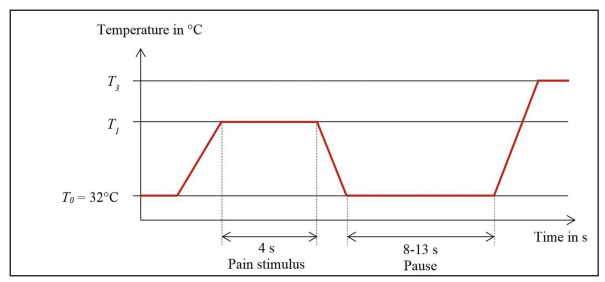

Fig. 3. An example of a $4 \mathrm{~s}$ pain stimulus that represents $T_{1}$ (pain threshold), and the subsequent pause.

(i.e., erotic and sport categories), 30 unpleasant (i.e., fear and disgust categories) and 30 neutral images were presented under the three pain levels. In total 90 image stimuli were presented with pain stimulation and 30 image stimuli were displayed without pain to every participant in each experimental phase. For each pain level and the baseline temperature all presented images were selected by stratified randomization out of the three categories (pleasant, unpleasant and neutral). The pleasant and unpleasant images were both high in arousal; the neutral images were low in arousal. 108 images were selected from the IAPS $[16]^{1}$. These emotional stimuli have normative ratings on affective valence and arousal. Moreover, these ratings have good stability and covary with physiological events $[4,15,17]$. These images have been utilized extensively in psychophysiological studies and affective computing research [2,21,23, 25, 27]. Furthermore, 72 images were selected from the EmoPicS $[30]^{2}$. The EmoPicS consists of photographic

1 The IAPS identification numbers for the pleasant images were: 1650, 2216, 4311, 4611, 4658, 4659, 4664, 4676, 4677, 4690, 4694, 4695, 4800, 4810, 5460, 5470, 5626, $5629,7502,8030,8080,8178,8179,8180,8185,8186,8191,8193,8210,8251,8300$, $8340,8341,8370,8499,8501$. The identification numbers for the unpleasant images were: $1050,1052,1113,1120,1201,1525,1932,2811,3150,3250,3400,3500,5972$, $6021,6022,6210,6212,6260,6312,6315,6415,6510,6530,6550,6570,6821,8480$, $8485,9050,9250,9254,9300,9600,9620,9622,9902,9910,9921$. The identification number for the neutral images were: 5471, 5731, 6150, 7002, 7009, 7025, 7030, 7034, 7035, 7036, 7038, 7040, 7041, 7050, 7052, 7053, 7055, 7056, 7057, 7059, 7090, 7100, $7130,7140,7150,7161,7170,7185,7233,7235,7493,7500,7547,7705$. Mean valence and arousal ratings across image sets were: pleasant images (valance: $\mathrm{M}=6.94$, arousal: $\mathrm{M}=6.40$ ), unpleasant images (valance: $\mathrm{M}=2.72$, arousal: $\mathrm{M}=6.42$ ), and neutral images (valance: $\mathrm{M}=5.03$, arousal: $\mathrm{M}=2.96$ ).

2 The EmoPicS identification numbers for the pleasant images were: 006, 008, 028, 043, 050, 052, 053, 055, 056, 057, 058, 059, 061, 062, 063, 064, 065, 066, 067, 069, $070,071,075,078$. The identification numbers for the unpleasant images were: 207 , 210, 211, 213, 214, 216, 219, 222, 229, 231, 232, 235, 238, 244, 250, 251, 252, 254, $321,325,326,329$. The identification numbers for the neutral images were: 123,125 , 127, 185, 188, 195, 196, 277, 281, 301, 302, 318, 335, 341, 342, 349, 354, 356, 365, $368,371,372,373,374,376,377$. Mean valence and arousal ratings across image sets were: pleasant images (valance: $\mathrm{M}=6.94$, arousal: $\mathrm{M}=5.57$ ), unpleasant images (valance: $\mathrm{M}=2.61$, arousal: $\mathrm{M}=6.26$ ), and neutral images (valance: $\mathrm{M}=5.02$, arousal: $\mathrm{M}=2.84)$. 
affective stimuli with different content and validated normative ratings which are expected to trigger primary motivational states as defined by the theoretical concept of motivational systems [15]. The EmoPicS was developed to serve as a supplement to IAPS. Each picture was presented on a computer screen for $6 \mathrm{~s}$ while the individual levels of pain were induced, and SCL, cardiac electrical activity, EMG activity and respiration were measured; moreover, video and audio signals were recorded. The order of the presentation was random.

Affective Sound Stimuli. Each image stimulus was accompanied by a sound stimulus. In the present experiment, the sound stimuli were selected to intensify emotional responses that would be induced by the image stimuli. To this end, sounds were carefully matched to image stimuli in regard to affective valence and arousal. For example, an image of a barking dog was accompanied by an aggressive growling. Each sound stimulus was presented along with the corresponding picture for $6 \mathrm{~s}$ over headphones.

\subsection{Measured Parameters}

Physiological measures and self-report were collected. Biosignal and event data were recorded via Social Signal Interpretation (SSI) [26] (see Fig. 4). SSI provides a flexible open source framework to apply on-the-fly signal processing and pattern recognition to extract higher-level information in real-time. Apart from physiological sensory, a wide range of devices are supported, including audiovisual sensory, motion capture suits, data gloves, pressure-sensitive mats, etc. A core task of SSI is the creation of multimodal databases. To this end, SSI supports the realization of complex multimodal recording setups, possibly distributed over several machines in a network and mechanisms to keep captured data in synchronization without time-stamping. An easy-to-use text-based interface allows users to set up dedicated systems for recording and analyzing multimodal signals without demanding any programming skills.

Biopotentials. g.MOBIlab+ (multi-purpose version) was utilized to acquire EMG and ECG, g.GSRsensor was used to measure SCL. Piezo-electric crystal sensor was utilized to record chest respiration waveforms (for further information: www.gtec.at/Products/Electrodes-and-Sensors/g.Sensors-Specs-Features). The following physiological parameters were measured.

SCL. Skin conductance is a measure of the conductivity of the skin, which especially increases if the skin becomes sweaty [7]. Two electrodes were positioned on the volar pads of the distal phalanges of the index and ring finger to measure SCL. Electrodermal activity is considered to be a sensitive indicator of the inner tension of an individual because sweat glands are innervated by the sympathetic branch of the autonomic nervous system (ANS). For instance, a rapid increase in skin conductance response can be reproduced within 1-3s by the exposure to a stress stimulus - e.g., emotional arousal or intense mental effort. 


\section{SenseEmotion experimental setup}

Heat pain stimulation and affective image stimuli with sound presentation
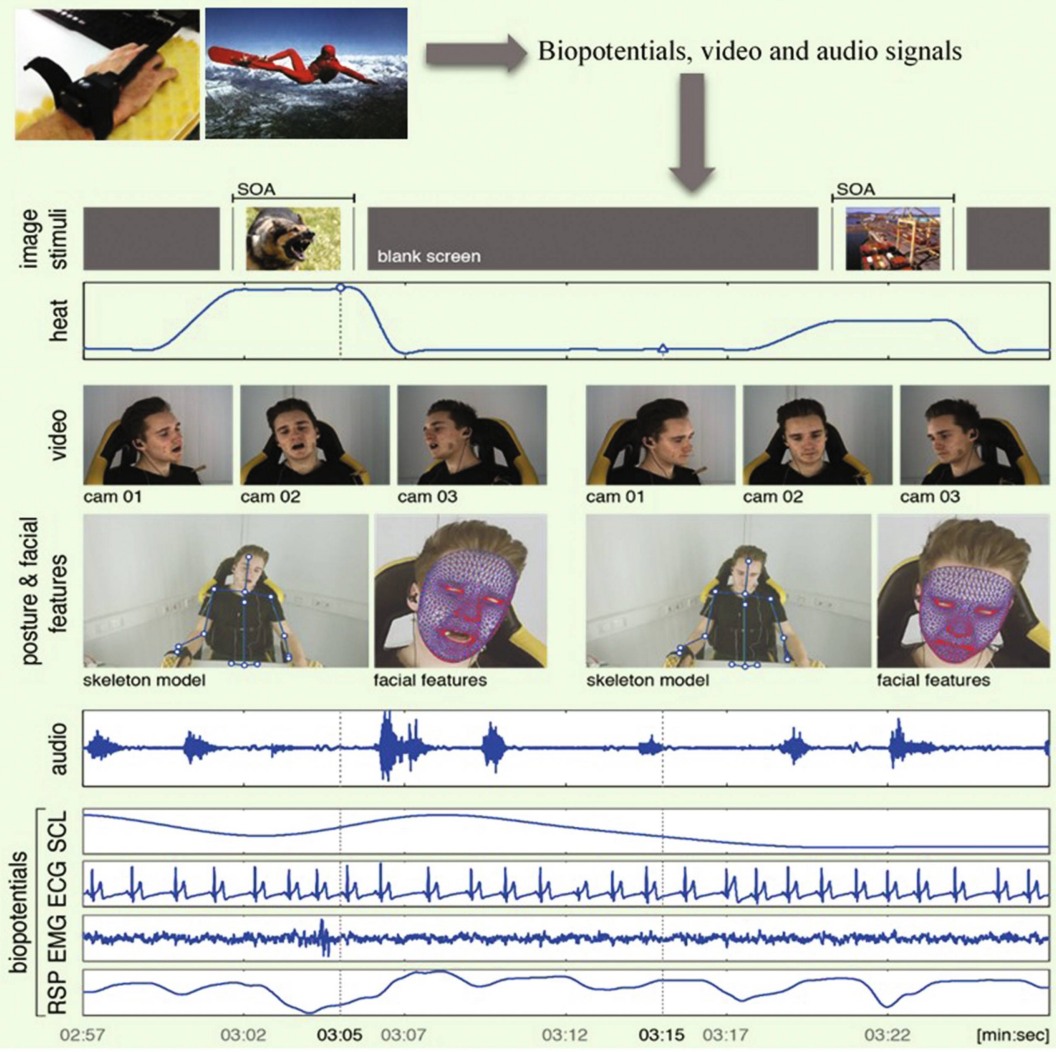

Fig. 4. Overview of the main experimental phase and the parameters that were measured. First row: the right affective stimulus was selected from the IAPS; the identification number of the image was 8186 . Second row: the left affective stimulus was selected from the EmoPicS; the identification number of the image was 325 . The right affective stimulus was selected from the IAPS; the identification number of the image was 7036 .

ECG. Three pregelled single $\mathrm{Ag} / \mathrm{AgCl}$ electrodes were utilized to measure the average cardiac action potential on the skin. One electrode was placed below the right clavicle (2nd interspace, right midclavicular line). The second electrode was placed on the left lower rib cage (8th interspace, left midclavicular line). The reference electrode was placed on the $\mathrm{C} 7$ spinous process. Common features of the ECG signal are heart rate, interbeat interval and heart rate variability (HRV). Heart rate reflects emotional activity [13]. In general, it has been used 
to distinguish positive from negative emotions. HRV refers to the oscillation of the interval between consecutive heartbeats. It is an indicator of mental effort and stress in adults.

EMG. Three pregelled single $\mathrm{Ag} / \mathrm{AgCl}$ electrodes were utilized to quantify muscle activity of the right upper trapezius muscle. Two electrodes were placed on a straight line from the spine of the 7 th cervical vertebra $(\mathrm{C} 7)$ to the lateral edge of acromion spanning the midpoint between the two landmarks [10]. The reference electrode was the same one that was used as reference for ECG. Electrical muscle activity indicates general psychophysiological arousal [6]. In particular, an increase in muscle tone reflects an increase in the activity of the sympathetic nervous system, while a decrease in somatomotor activity is mainly associated with parasympathetic arousal. The high level of muscle tension is an indicator of stress [7], which is also expected to occur under the experience of pain $[6,27]$.

RSP. Respiration sensor using an elastic belt system was thoracically worn by the participants over clothing. The most common measures of RSP are the rate and depth of breathing. Evidence for links between emotions and RSP suggests that different emotional states may give rise to different respiratory patterns [1]. Negative emotions can generally induce an irregular breathing pattern [13]. For example, RSP rate usually decreases with relaxation; however, shock exposure or tense situations may cause momentary RSP cessation. Furthermore, fast and deep breathing can be an indicator of emotional arousal such as excitement and joy [7]. Slow and deep breathing can indicate a relaxing state. Owing to the strong effect of RSP on heart rate, RSP is an interesting physiological signal to consider for affective computing both as a signal on its own and to investigate in conjunction with cardiac function [9].

Video Signals. A synchronized camera system was utilized to capture the faces of the participants (see Fig.4). The camera system consisted of three industrial cameras (iDS UI-3060CP-C-HQ), which were equipped with identical lenses (Tevidon 1.8/16). This particular camera system allowed the participants to move their heads freely ensuring that their faces were fully visible even in case of large out of plane rotations. One camera was placed directly in front of the participant and two at the side. The left and right cameras captured a frontal face in case the participant turned their head $45^{\circ}$ to the left or right, respectively. Each camera was connected to a dedicated recording computer via a USB 3.0 cable. Synchronization was realized by externally triggering the three cameras using the ChibiOS real time operation system running on the Arduino Due platform. The implemented setup was capable of constraining the temporal differences of the captured frames within tens of microseconds. Before the recording of participants, the researchers used a checkerboard pattern to calibrate the three cameras. When the recording started, the SSI software [26] first triggered the Arduino board. Then, the board sent triggering signals to all the cameras frequently at a pre-specified frame rate. To optimize the illumination condition, three large LED panels surrounding the participant were used. For the recordings the researchers set the frame resolution to $1600 \times 1200$, the frame rate to $60 \mathrm{fps}$ 
for the first 24 recording sessions and to 30 fps for the remaining 21 recordings. Exposure time was set to $15 \mathrm{~ms}$. To convert the raw data to accessible video files, the researchers first performed demosaicing using the built-in function in the OpenCV library and used the codec H.264 to compress the data losslessly. For missing frames due to data transfer failure they reconstructed these frames via temporal interpolation according to the camera time stamps. Additionally, the setup featured a Microsoft Kinect V2. This device was used to record color images at a resolution of $1920 \times 1080$ pixels at 30fps. The built in depth camera using a time-of-flight sensor was able to provide additional information about the participant. The researchers recorded skeleton data in 3D describing the position of 25 joints, head pose on three axes, 1347 face points in 3D and in $2 \mathrm{D}$ for projection on the color image and 17 facial action units at $30 \mathrm{~Hz}$.

Audio Signals. The audio recordings were performed using primarily a digital wireless headset microphone (Line6 XD-V75HS) in combination with a directional microphone (Rode M3). The wireless headset allows unconstrained head movements and records any sound produced by the participants. Typical sounds recorded during these experiments are breathing, moaning and sighing sounds. Meanwhile, the directional microphone records the ambient acoustic sounds. An additional audio stream was recorded using the Microsoft Kinect V2 integrated microphone. This microphone also records the ambient acoustic sounds. All recordings were performed with the sample rate set to $48 \mathrm{kHz}$. The three audio streams were synchronously recorded with the video and bio-physiological streams using the SSI framework [26].

Visual Analogue Scale. To assess the consistency of subjective criteria utilized to indicate $T_{1}$ and $T_{3}$ participants rated their pain intensity on the Visual Analogue Scale (VAS) immediately after the end of each experimental phase. The VAS consists of a $100 \mathrm{~mm}$ line whose anchors range from no pain sensation (score of 0) to the most intense pain sensation imaginable (score of 100) [31]. Hence, a higher score points out greater pain intensity. The VAS was administered as a paper-and-pencil measure. Participants were asked to mark on the VAS horizontal straight line the point that they felt best represented their pain intensity.

Self-Assessment Manikin. To verify whether image stimuli elicited the intended emotional states, participants rated their subjective reaction to the induction of affect using the Self-Assessment Manikin (SAM) [3,14] which measures the valence and arousal with viewing each picture. It was emphasized that the researchers were interested in personal feelings and that correct or wrong answers were not possible. The scale includes two sets of five pictographs showing affective valence (unpleasant-pleasant) and arousal (calm-excited). The pleasure scale depicts a smiling figure at one extreme and a frowning figure at the other. The arousal scale represents a sleeping figure at the calm end and an excited and wide-eyed figure at the other. Participants using the mid-point of each scale indicate feeling neither happy nor unhappy, or neither calm nor excited (i.e., neutral). Both scales yielded ratings between 1 and 9 for each dimension, with 
higher scores being associated with greater subjective pleasure and arousal (lower scores in pleasure dimension are associated with greater displeasure). Ratings were made during picture presentation after the end of each experimental phase. The paper-and-pencil version of the SAM scale was utilized for the present experiment. Participants were instructed to make a mark under the scale of each dimension.

\subsection{Procedure}

The experiments were conducted at the Emotion $\mathrm{Lab}^{3}$, Department of Psychosomatic Medicine and Psychotherapy, University of Ulm. First, the researchers summarized each aspect of the content and procedures of the experiment, and obtained informed consent. Each participant's health status was determined by a brief interview to define eligibility. If the participant was eligible, they were seated in a sound and light attenuated room. Then, pain stimulator and physiological sensors were applied. The sites were first slightly abraded with a skin preparation gel and decreased with alcohol for attaching the physiological sensors. Participants were acclimated to the experimental context while they completed several questionnaires. They were informed that no known risk was associated with the procedures of the experiment but they might feel temporary discomfort during skin preparation for the sensors and during heat pain stimulation. The researchers emphasized that the discomfort would be temporary and under participant's control because they would define their pain threshold and pain tolerance threshold. Additionally, participants were told that they were able to leave the experiment at any time by pressing the emergency stop button.

The first 25 participants began with the right arm; the rest of them began with the left arm. For both arms $T_{1}, T_{2}$ and $T_{3}$ pain levels were determined and finally both arms of each participant were stimulated with heat pain. The experiment was organized into two phases as shown in Fig. 5. Phase 1 involved calibration phase that lasted $15 \mathrm{~min}$. The main experimental phase involved inducing the individual pain levels while the participant was viewing the image presentation and listening to sound stimuli. The specific procedure was as follows: the main experimental phase began with a preparatory cue which stayed on the screen until the participant adjusted the volume of the headphones for listening to sound stimuli. Sound test was followed by instructions for the participant. Participants were informed that a series of images would be showed on the screen during which they would need to view each image the entire time and listen to the respective sound allowing themselves to experience the potential emotions evoked by the stimuli; they were also instructed that pain stimuli might be induced during the presentation. After explaining the procedure, the researchers left the experimental room and monitored the participants by video camera from a control room. The main experimental phase lasted approximately $30 \mathrm{~min}$. This phase was followed by an after-rating condition. During this condition, each participant rated the intensity of $T_{1}$ and $T_{3}$ on the VAS. They rated simultaneously

\footnotetext{
${ }^{3}$ http://www.uni-ulm.de/ $\sim$ hhoffman/emotions.
} 
valance and arousal of six affective stimuli that aimed to evoke positive, negative and neutral emotions using the SAM; they also rated three images from the three sets of emotions without pain stimulation. The after-rating condition began with a $6 \mathrm{~s}$ presentation of the to-be-rated picture and a $4 \mathrm{~s}$ induction of the pain stimulus, directly after which the rating was made. The rating period was 30s, allowing sufficient time for ratings. There was an interval of 20 min between Phase 1 and 2. The same process was followed for Phase 2. After the end of Phase 1 and 2, participants were requested to apply a cold compress to the area of heat pain stimulation for at least $5 \mathrm{~min}$. At the end of the experiment, sensors were removed and participants were debriefed and thanked.

Phase 1

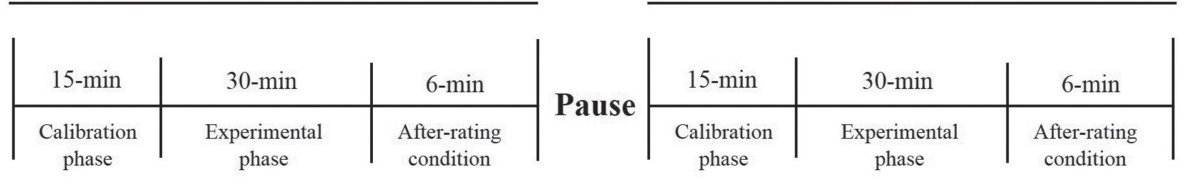

Fig. 5. Experimental procedure

\section{Conclusion}

The goal of the SenseEmotion project is the development of an automatic painand emotion-recognition system for the successful assessment and effective personalized management of pain in elderly. For this purpose, the present study was designed to gather multiple sources of information under heat pain stimulation and viewing image stimuli along with listening to sound stimuli aiming at emotion activation. The SenseEmotion Database consists of biopotentials (i.e., SCL, ECG, trapezius muscle EMG and RSP), video (facial expressions, skeleton data and head pose) and audio (paralinguistic information) signals. The data will be pre-analyzed with a variety of complex filter and decomposition techniques to extract and select meaningful feature patterns that will contribute to the highest recognition rate for pain- and emotion-recognition, pain quantification and differentiation between pain and emotion. Next steps will involve analyzing the data utilizing machine learning algorithms for offline and online analysis for painand emotion-recognition in real-time. Further, the researchers plan to advance the present study with the following key aspects:

1. The researchers will test and improve the generalizability with a complex pain model including phasic and tonic pain, heat pain and electrocutaneous stimulation.

2. The research group plans to test the automatic pain- and emotion-recognition system in clinical practice. One idea could be that the automatic recognition system would be tested in a post-operative setting of a care unit for people with dementia syndrome. 
To sum up, the SenseEmotion project advances towards its vision of an automatic pain- and emotion-recognition system that will facilitate pain assessment and management in older people in clinical and care home settings.

Acknowledgment. This paper is based on work done within the project SenseEmotion funded by the German Federal Ministry of Education and Research (BMBF).

\section{References}

1. Boiten, F.A., Frijda, N.H., Wientjes, C.J.E.: Emotions and respiratory patterns: review and critical analysis. Int. J. Psychophysiol. Official J. Int. Organ. Psychophysiol. 17(2), 103-128 (1994)

2. Bradley, M.M., Codispoti, M., Cuthbert, B.N., Lang, P.J.: Emotion and motivation I: defensive and appetitive reactions in picture processing. Emotion (Washington, D.C.) 1(3), 276-298 (2001)

3. Bradley, M.M., Lang, P.J.: Measuring emotion: the self-assessment manikin and the semantic differential. J. Behav. Ther. Exp. Psychiatry 25(1), 49-59 (1994)

4. Greenwald, M.K., Cook, E.W., Lang, P.J.: Affective judgment and psychophysiological response: dimensional covariation in the evaluation of pictorial stimuli. J. Psychophysiol. 3, 51-64 (1989)

5. Gruss, S.: Schmerzerkennung anhand psychophysiologischer Signale mithilfe maschineller Lerner. Dissertation, Universität Ulm (2015)

6. Gruss, S., Treister, R., Werner, P., Traue, H.C., Crawcour, S., Andrade, A., Walter, S.: Pain intensity recognition rates via biopotential feature patterns with support vector machines. PLoS ONE 10(10), 1-14 (2015)

7. Haag, A., Goronzy, S., Schaich, P., Williams, J.: Emotion recognition using biosensors: first steps towards an automatic system. In: André, E., Dybkjær, L., Minker, W., Heisterkamp, P. (eds.) ADS 2004. LNCS, vol. 3068, pp. 36-48. Springer, Heidelberg (2004). doi:10.1007/978-3-540-24842-2_4

8. Hammal, Z., Cohn, J.F.: Automatic detection of pain intensity. In: Proceedings of the 14th ACM International Conference on Multimodal Interaction, ICMI 2012, pp. 47-52. ACM, New York (2012)

9. Healey, J.: Physiological sensing of emotion. In: Calvo, R., D’Mello, S., Gratch, J., Kappas, A., (eds.) The Oxford Handbook of Affective Computing, pp. 204-216. Oxford University Press, New York (2015)

10. Jensen, C., Vasseljen, O., Westgaard, R.H.: The influence of electrode position on bipolar surface electromyogram recordings of the upper trapezius muscle. Eur. J. Appl. Physiol. 67(3), 266-273 (1993)

11. Kächele, M., Amirian, M., Thiam, P., Werner, P., Walter, S., Palm, G., Schwenker, F.: Adaptive confidence learning for the personalization of pain intensity estimation systems. Evolving Syst. 8(1), 71-83 (2017)

12. Kehlet, H.: Acute pain control and accelerated postoperative surgical recovery. Surg. Clin. North Am. 79(2), 431-443 (1999)

13. Kim, J., André, E.: Emotion recognition based on physiological changes in music listening. IEEE Trans. Pattern Anal. Mach. Intell. 30(12), 2067-2083 (2008)

14. Lang, P.J.: Behavioral treatment and bio-behavioral assessment: Computer applications. In: Sidowski, J.B., Johnson, J.H., Williams, T.A. (eds.) Technology in Mental Health Care Delivery Systems, pp. 119-137. Ablex Publishing, Norwood (1980) 
15. Lang, P.J.: The emotion probe: studies of motivation and attention. Am. Psychol. 50(5), 372-85 (1995)

16. Lang, P.J., Bradley, M.M., Cuthbert, B.N.: International affective picture system (IAPS): Affective ratings of pictures and instruction manual. Technical report A-8, University of Florida, Gainesville, FL (2008)

17. Lang, P.J., Greenwald, M.K., Bradley, M.M., Hamm, A.O.: Looking at pictures: affective, facial, visceral, and behavioral reactions. Psychophysiology 30(3), 261273 (1993)

18. Lautenbacher, S.: Schmerzmessung. In: Basler, H.D., Franz, C., Kröner-Herwig, B., Rehfisch, H.P. (eds.) Psychologische Schmerztherapie, pp. 271-288. Springer, Berlin (2004)

19. Limbrecht-Ecklundt, K., Werner, P., Traue, H.C., Walter, S.: Mimische Aktivität differenzierter Schmerzintensitäten. Korrelation der Merkmale von Facial Action Coding System und Elektromyografie. Der. Schmerz 30(3), 248-256 (2016)

20. McQuay, H., Moore, A., Justins, D.: Treating acute pain in hospital. Br. Med. J. 314(7093), 1531-1535 (1997)

21. Meagher, M.W., Arnau, R.C., Rhudy, J.L.: Pain and emotion: effects of affective picture modulation. Psychosom. Med. 63(1), 79-90 (2001)

22. Medoc advanced medical systems (2009)

23. Rhudy, J.L., Williams, A.E., McCabe, K.M., Nguyen, M.A., Rambo, P.: Affective modulation of nociception at spinal and supraspinal levels. Psychophysiology 42(5), 579-587 (2005)

24. Serpell, M.: Handbook of Pain Management. Springer, New York (2008)

25. Tan, J.W., Andrade, A.O., Li, H., Walter, S., Hrabal, D., Rukavina, S., LimbrechtEcklundt, K., Hoffman, H., Traue, H.C.: Recognition of intensive valence and arousal affective states via facial electromyographic activity in young and senior adults. PLoS ONE 11(1), 1-14 (2016)

26. Wagner, J., Lingenfelser, F., Baur, T., Damian, I., Kistler, F., André, E.: The social signal interpretation (SSI) framework: multimodal signal processing and recognition in real-time. In Proceedings of the 21st ACM International Conference on Multimedia - MM 2013, pp. 831-834. ACM Press, New York (2013)

27. Walter, S., Gruss, S., Ehleiter, H., Tan, J., Traue, H.C., Crawcour, S., Werner, P., Al-Hamadi, A., Andrade, A.O., Moreira da Silva, G.: The BioVid heat pain database - data for the advancement and systematic validation of an automated pain recognition system. In: 2013 IEEE International Conference on Cybernetics (CYBCONF), pp. 128-131. IEEE, June 2013

28. Walter, S., Gruss, S., Limbrecht-Ecklundt, K., Traue, H.C., Werner, P., Al-Hamadi, A., Diniz, N., Moreira, G., Andrade, A.O.: Automatic pain quantification using autonomic parameters. Psychol. Neurosci. 7(3), 363-380 (2014)

29. Werner, P., Al-Hamadi, A., Limbrecht-Ecklundt, K., Walter, S., Gruss, S., Traue, H.C.: Automatic pain assessment with facial activity descriptors. IEEE Trans. Affect. Comput. 99, 1-14 (2016)

30. Wessa, M., Kanske, P., Neumeister, P., Bode, K., Heissler, J., Schönfelder, S.: EmoPics: Subjektive und psychophysiologische Evaluation neuen Bildmaterials für die klinisch-bio-psychologische Forschung. Zeitschrift für Klinische Psychologie und Psychotherapie 39(Suppl. 1/11), 77 (2010)

31. Wewers, M.E., Lowe, N.K.: A critical review of visual analogue scales in the measurement of clinical phenomena. Res. Nurs. Health 13(4), 227-236 (1990)

32. Zwakhalen, S.M.G., Hamers, J.P.H., Abu-Saad, H.H., Berger, M.P.F.: Pain in elderly people with severe dementia: a systematic review of behavioural pain assessment tools. BMC Geriatr. 6(3), 1-15 (2006) 